\title{
ON STRONGLY FREELY DECOMPOSABLE AND INDUCED MAPS
}

\author{
Javier Camargo and Sergio Macías \\ Universidad Industrial de Santander, Colombia, Universidad Nacional \\ Autónoma de México, México
}

\begin{abstract}
Freely decomposable and strongly freely decomposable maps were introduced by G. R. Gordh and C. B. Hughes as a generalization of monotone maps with the property that these maps preserve local connectedness in inverse limits. We prove some relationships between $f$, $\mathcal{C}_{n}(f)$ and $2^{f}$, when $f, \mathcal{C}_{n}(f)$ or $2^{f}$ belong to the following classes of maps: Almost monotone, quasi-monotone, weakly monotone, freely decomposable or strongly freely decomposable.

We correct two corollaries formulated by Jaunusz J. Charatonik in "On feebly monotone and related classes of maps". We also present an alternative reformulation of these results.
\end{abstract}

\section{INTRODUCTION}

Freely decomposable and strongly freely decomposable maps were introduced by G. R. Gordh and C. B. Hughes in [10] as a generalization of monotone maps with the property that they preserve local connectedness in inverse limits. Strongly freely decomposable maps were studied by J. J. Charatonik under a different name, namely, feebly monotone maps ([4]).

The paper is divided in four sections. After the introduction and definitions, in Section 3 we construct a strongly freely decomposable map between irreducible continua such that it does not preserve the points of irreducibility (Example 3.1), this shows that [4, Corollary 5.5] is incorrect; we also present a quasi-monotone and confluent map $f:[0,1] \rightarrow[0,1]$ with point inverses having finitely many components such that $f(0)=f(1)=0$

2010 Mathematics Subject Classification. 54B20, 54E40, 54F15.

Key words and phrases. Confluent map, continua, freely decomposable map, irreducible continuum, local homeomorphism, monotone map, quasi-monotone map, strongly freely decomposable map. 
(Example 3.5), and a local homeomorphism $g: X \rightarrow Y$ between decomposable continua such that $X$ is irreducible between the points $a$ and $b$ but $Y$ is not irreducible between $g(a)$ and $g(b)$ (Corollary 3.8), this proves that [4, Corollary 5.6] is not true either; we give an alternative reformulation for [4, Corollary 5.5] (Theorem 3.3). In Section 4, we study relationships between $f, \mathcal{C}_{n}(f)$ and $2^{f}$, when $f, \mathcal{C}_{n}(f)$ or $2^{f}$ belong to the following classes of maps: Almost monotone, quasi-monotone, weakly monotone, freely decomposable or strongly freely decomposable. In particular, we prove that if $2^{f}$ or $\mathcal{C}_{n}(f)$ is almost monotone, then $f$ is almost monotone (Theorem 4.2), we show that the reverse implications are not true (Proposition 4.3, for $\mathcal{C}_{n}(f)$, and Remark 4.7, for $2^{f}$ ); we give an example of a map $f$ such that $f$ and $\mathcal{C}_{1}(f)$ are almost monotone but neither $2^{f}$ nor $\mathcal{C}_{n}(f)(n \geq 2)$ are strongly freely decomposable (Example 4.4); we prove the equivalence between the almost monotoneity and the strong free decomposability of $2^{f}$ and $\mathcal{C}_{n}(f)$, respectively, (Theorem 4.6); when the range of the map is locally connected we obtain a variety of equivalences between these induced maps (Corollary 4.10); and we present an example of a strongly freely decomposable map defined between locally connected continua such that its induced maps are not strongly freely decomposable (Example 4.11).

\section{Preliminaries}

If $X$ is a topological space, then given $A \subset X$, the interior of $A$ is denoted by $\operatorname{Int}_{X}(A)$, and its closure is denoted by $C l_{X}(A)$. The symbol $\mathbb{N}$ denotes the set of positive integers. Given a function $f: X \rightarrow Y$ between spaces, if $A$ is a subset of $X$, then $\left.f\right|_{A}$ denotes the restriction of $f$ to $A$.

A continuum is a nonempty compact, connected and metric space. A map is assumed to be a continuous function.

Let $f: X \rightarrow Y$ be a map between continua, we say that $f$ is:

- almost monotone if $f^{-1}(Q)$ is connected for every subcontinuum with nonempty interior $Q$ of $Y$.

- confluent provided that for each subcontinuum $Q$ of $Y$, if $D$ is a component of $f^{-1}(Q)$, then $f(D)=Q$.

- freely decomposable if whenever $A$ and $B$ are proper subcontinua of $Y$ such that $Y=A \cup B$, then there exist two proper subcontinua $A^{\prime}$ and $B^{\prime}$ of $X$, such that $X=A^{\prime} \cup B^{\prime}, A^{\prime} \subset f^{-1}(A)$ and $B^{\prime} \subset f^{-1}(B)$.

- local homeomorphism if for every point $x \in X$ there exists an open set $U$ such that $x \in U, f(U)$ is open and $\left.f\right|_{U}: U \rightarrow f(U)$ is a homeomorphism.

- monotone provided that $f^{-1}(Q)$ is connected, for each subcontinuum $Q$ of $Y$. 
- quasi-monotone if for every subcontinuum with nonempty interior $Q$ of $Y$, then $f^{-1}(Q)$ has a finite number of components and if $D$ is a component of $f^{-1}(Q)$, then $f(D)=Q$.

- strongly freely decomposable provided that whenever $A$ and $B$ are proper subcontinua of $Y$ such that $Y=A \cup B$, we obtain that $f^{-1}(A)$ and $f^{-1}(B)$ are connected ${ }^{1}$.

- weakly confluent provided that for each subcontinuum $Q$ in $Y$, there exists a component $D$ of $f^{-1}(Q)$ such that $f(D)=Q$.

- weakly monotone provided for every subcontinuum with nonempty interior $Q$ of $Y$, if $D$ is a component of $f^{-1}(Q)$, then $f(D)=Q$.

Clearly, each monotone map is almost monotone. Moreover, if $M=$ $C l_{\mathbb{R}^{2}}\left(\left\{\left(x, \sin \left(\frac{1}{x}\right)\right) \mid 0<x \leq 1\right\}\right)$ and $Y=X /\{(0,1),(0,-1)\}$, then the quotient map $q: X \rightarrow Y$ is an almost monotone map and $q$ is not monotone. $M$ is known as the topologist sine curve.

Observe that if $f$ is strongly freely decomposable, then $f$ is freely decomposable. Furthermore, it is not difficult to show that every almost monotone map is a strongly freely decomposable map. In [10, p. 139], it is shown that if $K$ is a nonconnected compactum, then the map $f: \operatorname{Sus}(K) \rightarrow$ $[-1,1]$, where $\operatorname{Sus}(K)$ is the topological suspension of $K$, defined by $f((k, t))=t$ is strongly freely decomposable and $f$ is not almost monotone.

Table I comprises all possible inclusions between the classes of maps on continua, which are considered here, an arrow means inclusion; i.e., the class of maps above is contained in the class of maps below. None of the reverse inclusions is true (for counterexamples see [15]).

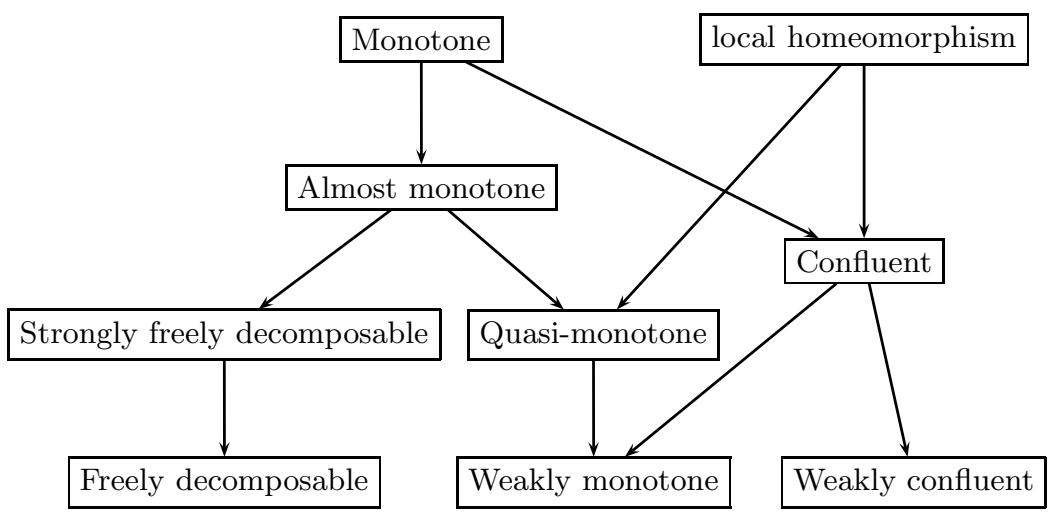

TABLE I

\footnotetext{
${ }^{1}$ Strongly freely decomposable maps are also known as feebly monotone maps in the literature.
} 
Given a continuum $X$, we define its hyperspaces as the following sets:

$$
\begin{aligned}
2^{X} & =\{A \subset X \mid A \text { is closed and nonempty }\} \\
\mathcal{C}_{n}(X) & =\left\{A \in 2^{X} \mid A \text { has at most } n \text { components }\right\}, n \in \mathbb{N} ; \\
\mathcal{F}_{n}(X) & =\left\{A \in 2^{X} \mid A \text { has at most } n \text { points }\right\}, n \in \mathbb{N} .
\end{aligned}
$$

We topologize these sets with the Hausdorff metric, $\mathcal{H}([16,(0.1)])$.

Let $A_{1}, \ldots, A_{m}$ be nonempty subsets of $X$. We define the set

$\left\langle A_{1}, \ldots, A_{m}\right\rangle=\left\{D \in 2^{X} \mid D \subset \cup_{i=1}^{m} A_{i}\right.$ and $D \cap A_{i} \neq \emptyset$ for each $\left.i\right\}$.

Note that this set is nonempty (for each $j \in\{1, \ldots, m\}$, let $a_{j} \in A_{j}$, then we have that $\left.\left\{a_{1}, \ldots, a_{m}\right\} \in\left\langle A_{1}, \ldots, A_{m}\right\rangle\right)$. It is known that the family $\left\{\left\langle U_{1}, \ldots, U_{l}\right\rangle \mid U_{1}, \ldots, U_{l}\right.$ are open subsets of $\left.X\right\}$ forms a basis for a topology on $2^{X}$ called Vietoris topology, $([16,(0.11)])$. It is well known that the Vietoris topology and the topology induced by the Hausdorff metric coincide ([16, $(0.13)])$. $\mathcal{C}_{n}(X)$.

To simplify notation, we write $\left\langle A_{1}, \ldots, A_{m}\right\rangle_{n}$ instead of $\left\langle A_{1}, \ldots, A_{m}\right\rangle \cap$

By definition, $\mathcal{C}_{n}(X)$ is a subspace of $2^{X}$, for each $n$. It is well known that $2^{X}$ is a continuum [16, (1.13)]. This is also true for $\mathcal{C}_{n}(X)$ (for $n=1$ see $[16,(1.13)]$ and for $n \geq 2$ see $[14,1.8 .12])$, and $\mathcal{F}_{n}(X)([14,1.8 .8])$ instead of $2^{X}$. A proof of the following lemma may be found at $[16,(1.48)]$.

LEMMA 2.1. The union function $\cup$ from $2^{2^{X}}$ onto $2^{X}$ given by $\cup(\mathcal{A})=$ $\cup\{A \mid A \in \mathcal{A}\}$ is a map.

The following result is Lemma 3.1 in [11].

Lemma 2.2. Let $\mathcal{K}$ be a subcontinuum of $2^{X}$ and let $K \in \mathcal{K}$. Then each component in $\cup \mathcal{K}$ intersects $K$.

Proposition 2.3. Let $X$ be a continuum and let $A, A_{1}, \ldots, A_{k}$ be subsets of $X$. Then:

(1) If $\langle A\rangle$ is a subcontinuum of $2^{X}$ or $\langle A\rangle_{n}$ is a subcontinuum of $\mathcal{C}_{n}(X)$, then $A$ is a subcontinuum of $X$;

(2) If $A_{1}, \ldots, A_{k}$ are subcontinua of $X$, then $\left\langle A_{1}, \ldots, A_{k}\right\rangle$ is a subcontinuum of $2^{X}$ and $\left\langle A_{1}, \ldots, A_{k}\right\rangle_{n}$ is a subcontinuum of $\mathcal{C}_{n}(X)$, whenever we have that $\left\langle A_{1}, \ldots, A_{k}\right\rangle_{n} \neq \emptyset$.

Proof. Note that (1) follows from Lemma 2.1.

To see (2), observe that $\left\langle A_{1}, \ldots, A_{k}\right\rangle$ is compact [12, Exercise 1.21]. Also note that $\cup_{i=1}^{k} A_{i} \in\left\langle A_{1}, \ldots, A_{k}\right\rangle$. Let $B \in\left\langle A_{1}, \ldots, A_{k}\right\rangle$. Then there exists an order arc $\alpha$ from $B$ to $\cup_{i=1}^{k} A_{i}$, by [16, (1.8)]. It is easy to see that $\alpha \subset$ $\left\langle A_{1}, \ldots, A_{k}\right\rangle$. Since $B$ is an arbitrary point of $\left\langle A_{1}, \ldots, A_{k}\right\rangle$, we have that $\left\langle A_{1}, \ldots, A_{k}\right\rangle$ is arcwise connected. Therefore, $\left\langle A_{1}, \ldots, A_{k}\right\rangle$ is a subcontinuum of $2^{X}$. 
The same argument shows that $\left\langle A_{1}, \ldots, A_{k}\right\rangle_{n}$ is a subcontinuum of $\mathcal{C}_{n}(X)$ if $\left\langle A_{1}, \ldots, A_{k}\right\rangle_{n} \neq \emptyset$.

\section{IRREDUCIBILITY AND STRONGLY FREELY DECOMPOSABLE MAPS}

In this section, we present an example showing that strongly freely decomposable maps do not preserve points of irreducibility. Then we prove that if a strongly freely decomposable map is defined between decomposable continua, then it preserves the points of irreducibility.

The following example shows that [4, Corollary 5.5] is not correct.

ExAmPlE 3.1. There exists a strongly freely decomposable map between irreducible continua such that it does not preserve the points of irreducibility.

Let $Y$ be an indecomposable subcontinuum of $\mathbb{R}^{2}$. Let $x$ and $z$ be two points of $Y$ such that $Y$ is irreducible between $x$ and $z$. Let $x^{\prime}$ be a point in the same composant of $x$ different from $x$. Define $X=(Y \times\{0\}) \cup\{(z, t) \mid 0 \leq$ $t \leq 1\} \cup(Y \times\{1\})$. Let $a=(x, 0)$ and $b=\left(x^{\prime}, 1\right)$. Observe that $X$ is a subcontinuum of $\mathbb{R}^{3}$ that is irreducible between $a$ and $b$. Now, define $f: X \rightarrow$ $Y$ by $f((y, t))=y$. Clearly, $f$ is continuous, $f(a) \neq f(b)$ and $Y$ is not irreducible between $f(a)$ and $f(b)$, because they belong to the same composant of $Y$. However, it is easy to see that $f$ is strongly freely decomposable (this also follows from Theorem 3.4).

Proposition 3.2. Let $X$ be an irreducible continuum between $a$ and $b$ and let $f: X \rightarrow Y$ be a strongly freely decomposable map. If $K$ is an irreducible subcontinuum of $Y$ between $f(a)$ and $f(b)$, then either $\operatorname{Int}_{Y}(K)=\emptyset$ or $K=Y$.

Proof. Since $X$ is irreducible and $f$ is a strongly freely decomposable map, $Y$ is irreducible between $f(a)$ and some other point [10, Theorem 14].

Suppose that $K$ is a subcontinuum of $Y$ such that $K$ is irreducible between $f(a)$ and $f(b), \operatorname{Int}_{Y}(K) \neq \emptyset$ and $K \neq Y$. Since $f(a)$ is a point of irreducibility

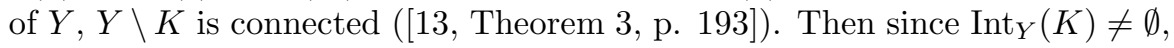
we have that $L=C l_{Y}(Y \backslash K)$ and $K$ are proper subcontinua of $Y$ such that $Y=L \cup K$. Thus, $f^{-1}(K)$ is connected, because $f$ is strongly freely decomposable. Hence, $f^{-1}(K)$ is a continuum. Note that $\{a, b\} \subset f^{-1}(K)$. Since $X$ is irreducible between $a$ and $b, f^{-1}(K)=X$, but this contradicts the fact that $K \neq Y$. Therefore, if $K$ is a subcontinuum of $Y$ that is irreducible between $f(a)$ and $f(b)$, then either $\operatorname{Int}_{Y}(K)=\emptyset$ or $K=Y$.

The next theorem shows that [4, Corollary 5.5] is true when the range of the map is a decomposable continuum.

THEOREM 3.3. Let $X$ be an irreducible continuum between $a$ and $b$. If $f: X \rightarrow Y$ is a strongly freely decomposable map, where $Y$ is a decomposable continuum, then $Y$ is irreducible between $f(a)$ and $f(b)$. 
Proof. Let $K$ be a subcontinuum of $Y$ that is irreducible between $f(a)$ and $f(b)$. By Proposition 3.2, either $\operatorname{Int}_{Y}(K)=\emptyset$ or $K=Y$. Suppose that $K \neq Y$. Then $\operatorname{Int}_{Y}(K)=\emptyset$. Since $Y$ is decomposable, there exist two proper subcontinua $M$ and $N$ of $Y$ such that $Y=M \cup N$. Since $K \subset Y, K \cap M \neq \emptyset$ or $K \cap N \neq \emptyset$. Assume that $K \cap M \neq \emptyset$. Since $\operatorname{Int}_{Y}(K)=\emptyset, K \cup M$ is a proper subcontinuum of $Y$. Clearly, $Y=(K \cup M) \cup N$. Hence, since $f$ is strongly freely decomposable, $f^{-1}(K \cup M)$ is a continuum. Note that $\{a, b\} \subset$ $f^{-1}(K \cup M)$. Since $X$ is irreducible between $a$ and $b, f^{-1}(K \cup M)=X$. This contradicts the fact that $K \cup M$ is a proper subcontinuum of $Y$. Thus, $K=Y$. Therefore, $Y$ is irreducible between $f(a)$ and $f(b)$.

Theorem 3.3 says that if there exists a strongly freely decomposable map $f: X \rightarrow Y$, where $X$ is a continuum that is irreducible between $a$ and $b$, and $Y$ is a continuum that is not irreducible between $f(a)$ and $f(b)$, then $Y$ must be indecomposable. Also, it is important to mention that in [10, Example 4] a freely decomposable map $f: X \rightarrow Y$ defined between decomposable continua is given such that $X$ is irreducible and $Y$ is not. Therefore, Theorem 3.3 is not true for freely decomposable maps.

The following result is [10, Theorem 13].

THEOREM 3.4. If $Y$ is an indecomposable continuum, then each map from a continuum onto $Y$ is strongly freely decomposable.

It is known that if $X$ is an irreducible continuum and $f$ is either a quasimonotone map or a confluent map such that for each $y$ in $Y, f^{-1}(y)$ has finitely many components, then $Y$ is an irreducible continuum ([8, Theorem $3]$ and [8, Corollary 6], respectively). Corollary 5.6 in [4] says that if $X$ is a continuum that is irreducible between $a$ and $b$ and $f$ is either quasi-monotone or confluent with point inverses having finitely many components, then $Y$ is a continuum that is irreducible between $f(a)$ and $f(b)$. The following example shows that Corollary 5.6 in [4] is not correct:

ExAmple 3.5. The map $f:[0,1] \rightarrow[0,1]$ given by $f(t)=1-\mid 2 t-$ $1 \mid$ is quasi-monotone and confluent with point inverses having finitely many components, but $f(0)=f(1)=0$.

However, [4, Corollary 5.6] also says that the above implication is true for local homeomorphisms. Although the map $f$ of Example 3.5 is not a local homeomorphism, the implication is not true, as we see in Theorem 3.7 and Corollary 3.8.

Let $\Sigma_{3}=\left\{\left(z_{n}\right)_{n=1}^{\infty} \in \prod_{n=1}^{\infty} S^{1} \mid z_{n+1}^{3}=z_{n}\right.$, for each postive integer $\left.n\right\}$. It follows from $[14,2.1 .19]$ that $\Sigma_{3}$ is an indecomposable continuum. $\Sigma_{3}$ is called the triadic solenoid.

LEMMA 3.6. If $f: \Sigma_{3} \rightarrow \Sigma_{3}$ is the map given by $f\left(\left(z_{n}\right)_{n=1}^{\infty}\right)=\left(z_{n}^{2}\right)_{n=1}^{\infty}$, for each $\left(z_{n}\right)_{n=1}^{\infty} \in \Sigma_{3}$, then $f$ is a local homemorphism. 
Proof. Note that $f$ is continuous by $[14,2.1 .46]$ and open by $[6$, Statement 10] and [9, Theorem 3]. We show that $\left|f^{-1}\left(\left(w_{n}\right)_{n=1}^{\infty}\right)\right|=2$ for each $\left(w_{n}\right)_{n=1}^{\infty} \in \Sigma_{3}$. Observe that $f$ is a surjective map, by [14, 2.1.48]. Let $\left(w_{n}\right)_{n=1}^{\infty} \in \Sigma_{3}$. Then there exists $\left(z_{n}\right)_{n=1}^{\infty} \in \Sigma_{3}$ such that $f\left(\left(z_{n}\right)_{n=1}^{\infty}\right)=$ $\left(w_{n}\right)_{n=1}^{\infty}$. Let $z_{1}^{\prime} \in S^{1}$ be such that $\left(z_{1}^{\prime}\right)^{2}=w_{1}$ and $z_{1}^{\prime} \neq z_{1}$. Suppose that there exist $z_{1}^{\prime}, \ldots, z_{k}^{\prime}$ such that $\left(z_{j+1}^{\prime}\right)^{3}=z_{j}^{\prime}$, for $j \in\{1, \ldots, k-1\}$, and $\left(z_{j}^{\prime}\right)^{2}=w_{j}$, for each $j \in\{2, \ldots, k\}$.

Note that it is not difficult to see that the following equations:

$$
x^{3}=z_{k}^{\prime}, \quad x^{2}=w_{k+1}, \text { where }\left(w_{k+1}\right)^{3}=\left(z_{k}^{\prime}\right)^{2}
$$

have only one solution. Let $z_{k+1}^{\prime}$ be such solution. Thus, by mathematical induction, there exists $\left(z_{n}^{\prime}\right)_{n=1}^{\infty}$ in $\Sigma_{3}$ such that $f\left(\left(z_{n}^{\prime}\right)_{n=1}^{\infty}\right)=\left(w_{n}\right)_{n=1}^{\infty}$. Since $z_{1}^{\prime} \neq z_{1},\left(z_{n}^{\prime}\right)_{n=1}^{\infty} \neq\left(z_{n}\right)_{n=1}^{\infty}$. Therefore, $\left|f^{-1}\left(\left(w_{n}\right)_{n=1}^{\infty}\right)\right| \geq 2$. Let $\left(x_{n}\right)_{n=1}^{\infty} \in$ $f^{-1}\left(\left(w_{n}\right)_{n=1}^{\infty}\right)$. Since $z_{1}$ and $z_{1}^{\prime}$ are the square roots of $w_{1}$, we have that either $x_{1}=z_{1}$ or $x_{1}=z_{1}^{\prime}$. Assume that $x_{1}=z_{1}$. We show that $\left(x_{n}\right)_{n=1}^{\infty}=$ $\left(z_{n}\right)_{n=1}^{\infty}$. Suppose that $x_{k} \neq z_{k}$ for some positive integer $k$. Since $\left(x_{k}\right)^{2}=$ $\left(z_{k}\right)^{2}=\left(z_{k}^{\prime}\right)^{2}=w_{k}$ and $z_{k} \neq z_{k}^{\prime}$, we have that $x_{k}=z_{k}^{\prime}$. Then $x_{1}=z_{1}^{\prime}$, this contradicts the fact that $x_{1}=z_{1}$ and $z_{1} \neq z_{1}^{\prime}$. Therefore, $\left(x_{n}\right)_{n=1}^{\infty}=\left(z_{n}\right)_{n=1}^{\infty}$ and $\left|f^{-1}\left(\left(w_{n}\right)_{n=1}^{\infty}\right)\right|=2$. Now, the lemma follows from [15, (4.27)].

Theorem 3.7. There exists a local homeomorphism $f: X \rightarrow Y$ between indecomposable continua such that $X$ is irreducible between a and b, but $Y$ is not irreducible between $f(a)$ and $f(b)$.

Proof. Let $X=Y=\Sigma_{3}$. Let $f: \Sigma_{3} \rightarrow \Sigma_{3}$ be a map defined by $f\left(\left(z_{n}\right)_{n=1}^{\infty}\right)=\left(z_{n}^{2}\right)_{n=1}^{\infty}$, for each $\left(z_{n}\right)_{n=1}^{\infty} \in \Sigma_{3}$. Note that $f$ is a local homeomorphism, by Lemma 3.6.

We claim that if $x=\left(x_{n}\right)_{n=1}^{\infty}$ and $y=\left(y_{n}\right)_{n=1}^{\infty}$ are the different points in $f^{-1}\left(\left(w_{n}\right)_{n=1}^{\infty}\right)$, for some $\left(w_{n}\right)_{n=1}^{\infty} \in \Sigma_{3}$, then $x_{n} \neq y_{n}$ for each positive integer $n$.

To prove this, suppose there exists a positive integer $k$ such that $x_{k}=y_{k}$. Clearly, $x_{l}=y_{l}$ for each $l \leq k$. We prove that $x_{k+1}=y_{k+1}$. Since $f(x)=f(y)$, $x_{k+1}^{2}=y_{k+1}^{2}$. Also, $x_{k+1}^{3}=x_{k}$ and $y_{k+1}^{3}=y_{k}$. Hence, $x_{k+1}^{3}=y_{k+1}^{3}$. Thus, it is not difficult to show that $x_{k+1}=y_{k+1}$. Therefore, $x=y$.

We assert that if $D$ is a proper subcontinuum of $\Sigma_{3}$, then $\left.f\right|_{D}: D \rightarrow f(D)$ is a homeomorphism.

To show this, note that, since $f$ is a closed map, we only need to prove that $\left.f\right|_{D}: D \rightarrow f(D)$ is one to one. Suppose that there exist two different points $x=\left(x_{n}\right)_{n=1}^{\infty}$ and $y=\left(y_{n}\right)_{n=1}^{\infty}$ of $D$ such that $f(x)=f(y)$. Hence, $x_{n} \neq y_{n}$ for any positive integer $n$, by our claim above. Given a positive integer $m$, let $f_{m}: \Sigma_{3} \rightarrow S^{1}$ be the projection defined by $f_{m}\left(\left(z_{n}\right)_{n=1}^{\infty}\right)=z_{m}$. Since $D$ is a proper subcontinuum of $\Sigma_{3}$, there exists an integer $k$ such that $f_{j}(D) \neq S^{1}$ for any $j \geq k,([14,2.1 .20])$. Observe that since $x_{k+1} \neq y_{k+1}$ and $x_{k+1}^{2}=y_{k+1}^{2}$, we have that $\operatorname{diam}\left(f_{k+1}(D)\right) \geq \pi$. Furthermore, $f_{k}(D)=\left(f_{k+1}(D)\right)^{3}$, by 
$[14,2.1 .6]$. Thus, $f_{k}(D)=S^{1}$, but this contradicts the fact that $f_{j}(D) \neq S^{1}$ for any $j \geq k$. Therefore, $\left.f\right|_{D}: D \rightarrow f(D)$ is a homeomorphism.

Finally, let $a$ and $b$ be different points of $\Sigma_{3}$ such that $f(a)=f(b)$. Thus, $\Sigma_{3}$ is irreducible between $a$ and $b$, by our assertion above. Clearly, $\Sigma_{3}$ is not irreducible between $f(a)$ and $f(b)$.

The following corollary shows that there exists a local homeomorphism between decomposable continua that does not preserve points of irreducibility.

COROLlary 3.8. There exists a local homeomorphism $g: X \rightarrow Y$ such that $g$ is defined between decomposable continua, $X$ is irreducible between a and $b$, and $Y$ is not irreducible between $g(a)$ and $g(b)$.

Proof. Let $f: \Sigma_{3} \rightarrow \Sigma_{3}$ be given by $f\left(\left(z_{n}\right)_{n=1}^{\infty}\right)=\left(z_{n}^{2}\right)_{n=1}^{\infty}$, for each $\left(z_{n}\right)_{n=1}^{\infty} \in \Sigma_{3}$. Let $a$ and $b$ be different points of $\Sigma_{3}$ such that $f(a)=f(b)=c$. Observe that $\Sigma_{3}$ is irreducible between $a$ and $b$, by the proof of Theorem 3.7. Also note that $f$ is a local homeomorphism, by Lemma 3.6. In fact, by the proof of Lemma 3.6, we have that $\left|f^{-1}(w)\right|=2$ for each $w \in \Sigma_{3}$.

Let $\alpha=\overline{a_{1} a_{2}}, \beta=\overline{b_{1} b_{2}}$ and $\gamma=\overline{c_{1}, c_{2}}$ be arcs such that $\alpha, \beta, \gamma$ and $\Sigma_{3}$ are pairwise disjoint continua. Let $\psi:\left\{a_{1}, b_{1}\right\} \rightarrow\{a, b\}$ be given by $\psi\left(a_{1}\right)=a$ and $\psi\left(b_{1}\right)=b$. Let $X=(\alpha \cup \beta) \cup \cup_{\psi} \Sigma_{3}$ (the adjunction space) and let $Y=\left(\gamma \cup \Sigma_{3}\right) /\left\{c_{1}, c\right\}$.

Let $g: X \rightarrow Y$ be a map defined by $g(z)=f(z)$ if $z \in \Sigma_{3}$ and such that, $\left.g\right|_{\alpha}: \alpha \rightarrow \gamma$ and $\left.g\right|_{\beta}: \beta \rightarrow \gamma$ are homeomorphisms. Note that $\left|g^{-1}(w)\right|=2$ for each $w \in Y$. Furthermore, since $\left.g\right|_{\Sigma_{3}}=f,\left.g\right|_{\alpha}$ and $\left.g\right|_{\beta}$ are open, it is not difficult to show that $g$ is an open map. Therefore, $g$ is a local homeomorphism $([15,(4.27)])$.

Since $\Sigma_{3}$ is irreducible between $a$ and $b$, it is easy to see that $X$ is irreducible between $a_{2}$ and $b_{2}$. By construction, $g\left(a_{2}\right)=g\left(b_{2}\right)$. Therefore, $Y$ is not irreducible between $g\left(a_{2}\right)$ and $g\left(b_{2}\right)$.

\section{INDUCED MAPS}

Let $f: X \rightarrow Y$ be a map between continua. Then the function $2^{f}: 2^{X} \rightarrow$ $2^{Y}$ given by $2^{f}(A)=f(A)$ for each $A \in 2^{X}$, is called the induced map between $2^{X}$ and $2^{Y}$. It is known that $2^{f}$ is a map $([14,1.8 .22])$. The map $\mathcal{C}_{n}(f): \mathcal{C}_{n}(X) \rightarrow \mathcal{C}_{n}(Y)$ given by $\mathcal{C}_{n}(f)=\left.2^{f}\right|_{\mathcal{C}_{n}(X)}$ is called the induced map between the $n$-fold hyperspaces $\mathcal{C}_{n}(X)$ and $\mathcal{C}_{n}(Y)$.

The following proposition is used later.

Proposition 4.1. Let $f: X \rightarrow Y$ be a map between continua and let $D$ be a subcontinuum of $Y$. If $E$ is a component of $f^{-1}(D)$, then $\langle E\rangle$ is a component of $\left(2^{f}\right)^{-1}(\langle D\rangle)$ and $\langle E\rangle_{n}$ is a component of $\mathcal{C}_{n}(f)^{-1}\left(\langle D\rangle_{n}\right)$, for each $n \in \mathbb{N}$. 
Proof. The case of the map $\mathcal{C}_{n}(f)$ is proved in [1, Proposition 3.3]. We show the case of the map $2^{f}$.

Let $f: X \rightarrow Y$ be a map between continua, let $D$ be a subcontinuum of $Y$ and let $E$ be a component of $f^{-1}(D)$. We show that $\langle E\rangle$ is a component of $\left(2^{f}\right)^{-1}(\langle D\rangle)$. Observe that $\langle E\rangle$ is a continuum, by Proposition 2.3, and $2^{f}(\langle E\rangle) \subset\langle D\rangle$. Assume that there exists a subcontinuum $\mathcal{A}$ of $2^{X}$ such that $\langle E\rangle \subset \mathcal{A}$ and $2^{f}(\mathcal{A}) \subset\langle D\rangle$. Since $E \in \mathcal{A}, \cup \mathcal{A}$ is connected, by Lemma 2.2. Hence, $E=\cup \mathcal{A}$, because $E$ is a component to $f^{-1}(D)$. Thus, if $B \in \mathcal{A}$, then $B \subset E$. Therefore, $\langle E\rangle=\mathcal{A}$ and $\langle E\rangle$ is a component of $\left(2^{f}\right)^{-1}(\langle D\rangle)$.

TheOREM 4.2. Let $f: X \rightarrow Y$ be a map between continua and let $n \in$ $\mathbb{N}$. If either $2^{f}$ or $\mathcal{C}_{n}(f)$ is an almost monotone map, then $f$ is also almost monotone.

Proof. Let $f: X \rightarrow Y$ be a map between continua such that $2^{f}$ is almost monotone. Let $A$ be a subcontinuum of $Y$ such that $\operatorname{Int}_{Y}(A) \neq \emptyset$. Then $\langle A\rangle$ is a subcontinuum $2^{Y}$, by Proposition 2.3. Moreover, $\langle A\rangle$ has nonempty interior, because $\left\langle\operatorname{Int}_{Y}(A)\right\rangle \subset\langle A\rangle$. Since $2^{f}$ is almost monotone, $\left(2^{f}\right)^{-1}(\langle A\rangle)$ is connected. It is not difficult to see that $\left(2^{f}\right)^{-1}(\langle A\rangle)=\left\langle f^{-1}(A)\right\rangle$. Hence, $f^{-1}(A)$ is connected, by Proposition 2.3. Therefore, $f$ is almost monotone.

It is easy to show, using the same argument, that if $\mathcal{C}_{n}(f)$ is an almost monotone map, then $f$ is almost monotone.

The next proposition shows that the converse of Theorem 4.2 is not true for the induced map $\mathcal{C}_{n}(f)$ for any $n \in \mathbb{N}$ (for the converse of Theorem 4.2 with respect to $2^{f}$ see Remark 4.7).

Proposition 4.3. There exists an almost monotone map $f$ such that $\mathcal{C}_{n}(f)$ is not strongly freely decomposable for any $n \in \mathbb{N}$. Hence, $\mathcal{C}_{n}(f)$ is not almost monotone, for any $n \in \mathbb{N}$.

Proof. Let $X=J \cup R$, where $J=\{(0, y) \mid-1 \leq y \leq 3\}$ and $R=$ $\left\{\left(x, \sin \left(\frac{1}{x}\right)\right) \mid 0<x \leq \frac{2}{\pi}\right\}$. Define $Y=X / \sim$, where $\left(x_{0}, y_{0}\right) \sim\left(x_{1}, y_{1}\right)$ if and only if $x_{0}=x_{1}=0$ and $e^{\pi y_{0} i}=e^{\pi y_{1} i}$. Let $f: X \rightarrow Y$ be the quotient map. Note that $f$ is an almost monotone map. Observe that $Y=S \cup R^{\prime}$, where $S=f(J)$ and $R^{\prime}=f(R)$. Without loss of generality we assume that $S=\left\{x \in \mathbb{R}^{2} \mid\|x\|=1\right\}$ (see Figure 1 ).

We prove that $\mathcal{C}_{n}(f)$ in not strongly freely decomposable for any $n \in$ $\mathbb{N}$. First, we assume that $n=1$. Let $L_{1}=\left\{(0, y) \mid \frac{1}{2} \leq y \leq \frac{3}{2}\right\}$, let $L_{2}=\left\{(0, y) \mid \frac{5}{2} \leq y \leq 3\right\}$, and let $L_{3}=\left\{(0, y) \mid-1 \leq y \leq-\frac{1}{2}\right\}$. Define $A=f\left(L_{1}\right)=\left\{e^{\pi i t} \in S \mid \frac{1}{2} \leq t \leq \frac{3}{2}\right\}$. Let $\mathcal{A}=\langle A\rangle_{1}$. Note that, by Proposition $2.3, \mathcal{A}$ is a proper subcontinuum of $\mathcal{C}_{1}(Y)$.

Now, we show that $\operatorname{Int}_{\mathcal{C}_{1}(Y)}(\mathcal{A}) \neq \emptyset$. To this end, let $p=f((0,-1)) \in S$ and let $W=\left\{x \in \mathbb{R}^{2} \mid\|x-p\|<1\right\}$. It is not difficult to show that there exist three open subsets $U_{1}, U_{2}$ and $U_{3}$ of $\mathbb{R}^{2}$ such that: 
(1) $U_{1} \cup U_{2} \cup U_{3} \subset W$;

(2) $U_{i}$ is connected for each $i \in\{1,2,3\}$;

(3) $p \in U_{2} \backslash\left(U_{1} \cup U_{3}\right)$ and $U_{1} \cap U_{3}=\emptyset$;

(4) $\left(U_{1} \cup U_{2} \cup U_{3}\right) \cap A$ is connected.

Observe that if $E \in\left\langle U_{1}, U_{2}, U_{3}\right\rangle_{1}$, then $E \subset\left(\cup_{i=1}^{3} U_{i}\right) \cap A$. Hence, $\left\langle U_{1}, U_{2}, U_{3}\right\rangle_{1} \subset \mathcal{A}$. Therefore, $\operatorname{Int}_{\mathcal{C}_{1}(Y)}(\mathcal{A}) \neq \emptyset$. Let $\mathcal{B}=C l_{\mathcal{C}_{1}(Y)}\left(\mathcal{C}_{1}(Y) \backslash \mathcal{A}\right)$. Clearly, $\mathcal{B}$ is compact and, since $\operatorname{Int}_{\mathcal{C}_{1}(Y)}(\mathcal{A}) \neq \emptyset, \mathcal{B} \neq \mathcal{C}_{1}(Y)$. Furthermore, if $B \in \mathcal{C}_{1}(Y) \backslash \mathcal{A}$, then there exists an order arc $\alpha$ form $B$ to $Y([16,(1.8)])$. Note that $\alpha \subset \mathcal{C}_{1}(Y) \backslash \mathcal{A}$. Hence, $\mathcal{C}_{1}(Y) \backslash \mathcal{A}$ is arcwise connected. Thus, $\mathcal{B}$ is a proper subcontinuum of $\mathcal{C}_{1}(Y)$. Clearly, $\mathcal{C}_{1}(Y)=\mathcal{A} \cup \mathcal{B}$.

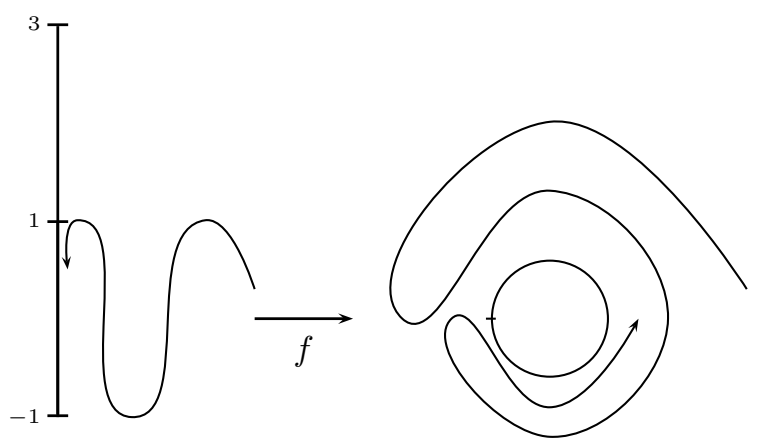

Figure 1

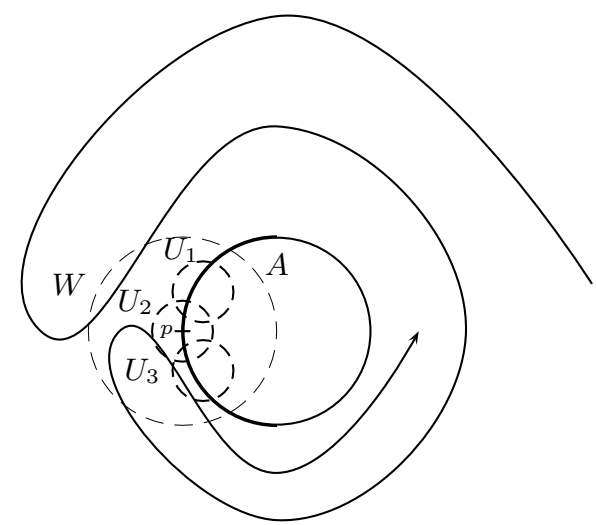

Figure 2 
We show that $\mathcal{C}_{1}(f)^{-1}(\mathcal{A})$ is not connected. Since $L_{1}, L_{2}$ and $L_{3}$ are the components of $f^{-1}(A)$, we have that $\left\langle L_{1}\right\rangle_{1},\left\langle L_{2}\right\rangle_{1}$ and $\left\langle L_{3}\right\rangle_{1}$ are components of $\mathcal{C}_{1}(f)^{-1}(\mathcal{A})$, by Proposition 4.1. Therefore, $\mathcal{C}_{1}(f)^{-1}(\mathcal{A})$ is not connected, and $\mathcal{C}_{1}(f)$ is not strongly freely decomposable.

Next, suppose $n \geq 2$. Let $L_{1}, L_{2}, L_{3}, A, W, U_{1}, U_{2}$ and $U_{3}$ be as before. Let $L_{4}=\left\{\left(x, \sin \left(\frac{1}{x}\right)\right) \mid \frac{1}{\pi} \leq x \leq \frac{2}{\pi}\right\}$ and let $A^{\prime}=f\left(L_{4}\right)$. Then $\mathcal{A}=\left\langle A, A^{\prime}\right\rangle_{n}$ is a subcontinuum of $\mathcal{C}_{n}(Y)$, by Proposition 2.3.

We show that $\mathcal{A}$ has nonempty interior. Let $V_{1}, V_{2}, \ldots, V_{n-1}$ be open, pairwise disjoint and connected subsets of $Y \backslash C l_{Y}(W)$ such that $\cup_{i=1}^{n-1} V_{i} \subset A^{\prime}$. Observe that if $E \in\left\langle U_{1}, U_{2}, U_{3}, V_{1}, \ldots, V_{n-1}\right\rangle_{n}$, then there exists a component $E_{0}$ of $E$ such that $E_{0} \subset A$ and $E \backslash E_{0} \subset \cup_{i=1}^{n-1} V_{i} \subset A^{\prime}$. Therefore, $\left\langle U_{1}, U_{2}, U_{3}, V_{1}, \ldots, V_{n-1}\right\rangle_{n} \subset \mathcal{A}$ and $\operatorname{Int}_{\mathcal{C}_{n}(Y)}(\mathcal{A}) \neq \emptyset$.

Let $\mathcal{B}=C_{\mathcal{C}_{n}(Y)}\left(\mathcal{C}_{n}(Y) \backslash \mathcal{A}\right)$. With a proof similar to the case $n=1$, we have that $\mathcal{B}$ is a proper subcontinuum of $\mathcal{C}_{n}(Y)$. In this case, we also have that $\mathcal{C}_{n}(Y)=\mathcal{A} \cup \mathcal{B}$.

Observe that $\left\langle L_{1}, L_{4}\right\rangle_{n}$ is a continuum by Proposition 2.3. Also note that $f^{-1}(A)=L_{1} \cup L_{2} \cup L_{3}$ and $f^{-1}\left(A^{\prime}\right)=L_{4}$. Hence, $\left\langle L_{1}, L_{4}\right\rangle_{n} \subset \mathcal{C}_{n}(f)^{-1}(\mathcal{A})$. In fact, $\left\langle L_{1}, L_{4}\right\rangle_{n}$ is an open and closed subset of $\mathcal{C}_{n}(f)^{-1}(\mathcal{A})$. We already know that $\left\langle L_{1}, L_{4}\right\rangle_{n}$ is closed. Let $D \in\left\langle L_{1}, L_{4}\right\rangle_{n}$ and let $\varepsilon>0$ be such that $\varepsilon<\frac{1}{2}$. Thus, clearly, if $E \in \mathcal{C}_{n}(f)^{-1}(\mathcal{A})$ and $\mathcal{H}(D, E)<\varepsilon$, then $E \in\left\langle L_{1}, L_{4}\right\rangle_{n}$ $\left(E \cap\left(L_{2} \cup L_{3}\right)=\emptyset\right)$. Hence, $\left\langle L_{1}, L_{4}\right\rangle_{n}$ is open in $\mathcal{C}_{n}(f)^{-1}(\mathcal{A})$. Therefore, $\mathcal{C}_{n}(f)^{-1}(\mathcal{A})$ is not connected, and $\mathcal{C}_{n}(f)$ is not strongly freely decomposable.

The map defined in the following example is taken from [7, Example 4.12, p. 138].

EXAMPLE 4.4. There exists a map $f: X \rightarrow Y$ between continua such that both $f$ and $\mathcal{C}_{1}(f)$ are almost monotone and neither $\mathcal{C}_{n}(f)$ nor $2^{f}$ is strongly freely decomposable, for any $n \geq 2$.

Define $H$ and $S$ in polar coordinates $(r, \theta)$ by:

$$
H=\left\{(r, \theta) \mid r=1+\theta^{-1} \text { and } \theta \in[1, \infty)\right\} \text { and } S=\{(1, \theta) \mid \theta \in[0,2 \pi]\} .
$$

Let $X=H \cup S$. Consider the equivalence relation on $X$ given by: $(r, \theta) \sim$ $\left(r^{\prime}, \theta^{\prime}\right)$ if and only if $(r, \theta)=\left(r^{\prime}, \theta^{\prime}\right)$ or, $r=r^{\prime}=1$ and $\theta=\theta^{\prime}+\pi$. Denote by $Y$ the quotient space and let $f: X \rightarrow Y$ be the quotient map.

We show that $\mathcal{C}_{1}(f)$ is almost monotone. Let $\mathcal{D}$ be a subcontinuum of $\mathcal{C}_{1}(Y)$ such that $\operatorname{Int}_{\mathcal{C}_{1}(Y)}(\mathcal{D}) \neq \emptyset$. It is not difficult to prove that there exists a point $D \in \mathcal{D}$ such that $D \subset f(H)$. Hence, either $\mathcal{D} \subset f(H)$ or, both $\mathcal{D} \cap f(H) \neq \emptyset$ and $\mathcal{D} \cap f(S) \neq \emptyset$. Thus, $\mathcal{C}_{1}(f)^{-1}(\mathcal{D})$ is connected, by cases $(1)$ and (3) from [7, Example 4.12, p. 139]. Therefore, $\mathcal{C}_{1}(f)$ is almost monotone.

Let $p=f((2,1))$ in $Y$. Since $Y$ is locally connected at $p$, there exists a subcontinuum $K$ of $Y$ such that $p \in \operatorname{Int}_{Y}(K) \subset K \subset Y \backslash f(S)$. Let $\mathcal{K}=\langle K\rangle \cup \mathcal{F}_{1}(Y)$. Clearly, $\mathcal{K}$ is a subcontinuum of $2^{Y}$ and $\operatorname{Int}_{2^{Y}}(\mathcal{K}) \neq$ 
$\emptyset$. Moreover, $\left(2^{f}\right)^{-1}(\mathcal{K})$ has two components: $\mathcal{F}_{1}(X) \cup\left\langle f^{-1}(K)\right\rangle$ and $\{\{(1, \theta),(1, \theta+\pi)\} \mid \theta \in[0, \pi]\}$. Thus, $\left(2^{f}\right)^{-1}(\mathcal{K})$ is not connected. Finally, it is not difficult to see that $2^{Y} \backslash \mathcal{K}$ is connected. Hence, $\mathcal{L}=C l_{2^{Y}}\left(2^{Y} \backslash \mathcal{K}\right)$ is a proper subcontinuum of $2^{Y}$. Clearly, $2^{Y}=\mathcal{K} \cup \mathcal{L}$. Therefore, $2^{f}$ is not strongly freely decomposable. Note that if $n \geq 2$, then we can take $\mathcal{K}=\left(\langle K\rangle_{n}\right) \cup \mathcal{F}_{1}(Y)$ and the same argument shows that $\mathcal{C}_{n}(f)$ is not strongly freely decomposable.

Question 4.1. Let $f: X \rightarrow Y$ be a map between continua. If $2^{f}$ is almost monotone, then does it follow that $\mathcal{C}_{n}(f)$ is almost monotone, for some $n \in \mathbb{N}$ ?

REMARK 4.5. Observe that, by Corollary 4.10, the answer to Question 4.1 is positive for maps whose range is locally connected.

Theorem 4.6. Let $f: X \rightarrow Y$ be a map between continua and let $n \in \mathbb{N}$. Then:

(1) $\mathcal{C}_{n}(f)$ is almost monotone if and only if $\mathcal{C}_{n}(f)$ is strongly freely decomposable;

(2) $2^{f}$ is almost monotone if and only if $2^{f}$ is strongly freely decomposable.

Proof. Since every almost monotone map is strongly freely decomposable, we have that the almost monotoneity of $\mathcal{C}_{n}(f)$ and $2^{f}$ implies their strong free decomposability. Since $\mathcal{C}_{n}(Y)$ and $2^{Y}$ are unicoherent for each $n \in \mathbb{N}$, by $[14,6.2 .4]$ and $[16,(1.176)]$, respectively, the converse implications follow from [2, Theorem 4.2].

REMARK 4.7. Note that, by Theorem 4.6, the map $f$ of Example 4.4 is an almost monotone map such that $2^{f}$ is not almost monotone.

The next result follows from Theorems 4.2 and 4.6.

Corollary 4.8. Let $f: X \rightarrow Y$ be a map between continua and let $n \in \mathbb{N}$. If either $2^{f}$ or $\mathcal{C}_{n}(f)$ is strongly freely decomposable, then $f$ is an almost monotone map.

Now we consider maps whose range are locally connected continua.

THEOREM 4.9. Let $f: X \rightarrow Y$ be a map between continua, where $Y$ is locally connected and let $n \in \mathbb{N}$. The following are equivalent:

(1) $\mathcal{C}_{n}(f)$ is freely decomposable;

(2) $\mathcal{C}_{n}(f)$ is monotone;

(3) $2^{f}$ is freely decomposable;

(4) $2^{f}$ is monotone;

(5) $f$ is monotone.

Proof. Suppose $\mathcal{C}_{n}(f)$ is freely decomposable, we prove that $\mathcal{C}_{n}(f)$ is monotone. Since $Y$ is locally connected, $\mathcal{C}_{n}(Y)$ is locally connected, by [14, 6.1.4]. Since $\mathcal{C}_{n}(Y)$ is colocally connected $([14,6.3 .1])$, it follows easily that $\mathcal{C}_{n}(Y) \backslash\{A\}$ is connected, for each $A \in \mathcal{C}_{n}(Y)$. Thus, $\mathcal{C}_{n}(f)^{-1}(A)$ is connected, 
by [10, Lemma 3]. Therefore, $\mathcal{C}_{n}(f)$ is monotone. Recall that the map $\mathcal{C}_{n}(f)$ is monotone if and only if $f$ is monotone, by [5, Theorem 4]. Hence, since every monotone map is freely decomposable, we have that (1), (2) and (5) are equivalent.

With a similar argument to the one given above, we prove that if $2^{f}$ is freely decomposable, then $2^{f}$ is monotone. Also recall that $2^{f}$ is monotone if and only if $f$ is monotone ([11, Theorem 3.2]). Thus, again since every monotone map is freely decomposable, we have that (3), (4) and (5) are equivalent.

Since every monotone map is strongly freely decomposable and every strongly freely decomposable map is freely decomposable, we have that the next corollary is a consequence of Theorems 4.9 and 4.6 .

Corollary 4.10. Let $f: X \rightarrow Y$ be a map between continua, where $Y$ is locally connected and let $n \in \mathbb{N}$. The following are equivalent:

(1) $\mathcal{C}_{n}(f)$ is freely decomposable;

(2) $\mathcal{C}_{n}(f)$ is strongly freely decomposable;

(3) $\mathcal{C}_{n}(f)$ is almost monotone;

(4) $\mathcal{C}_{n}(f)$ is monotone;

(5) $2^{f}$ is freely decomposable;

(6) $2^{f}$ is strongly freely decomposable;

(7) $2^{f}$ is almost monotone;

(8) $2^{f}$ is monotone;

(9) $f$ is monotone.

In the next example, we give a strongly freely decomposable map $f$ such that neither $2^{f}$ nor $\mathcal{C}_{n}(f)$ is strongly freely decomposable, for any $n \in \mathbb{N}$, not even, when $f$ is defined between locally connected continua.

EXAMPLE 4.11. There exists a strongly freely decomposable map between locally connected continua $f$ such that neither $2^{f}$ nor $\mathcal{C}_{n}(f)$ is strongly freely decomposable, for any $n \in \mathbb{N}$.

We denote $S^{1}=\left\{(x, y) \in \mathbb{R}^{2} \mid x^{2}+y^{2}=1\right\}$. Let $f: S^{1} \rightarrow[-1,1]$ be the map defined by $f((x, y))=x$. Clearly, $f$ is strongly freely decomposable and it is not montone. Hence, neither $\mathcal{C}_{n}(f)$ nor $2^{f}$ is freely decomposable, by Corollary 4.10 .

\section{REFERENCES}

[1] J. Camargo, Some relationships between induced mappings, Topology Appl. 157 (2010), 2038-2047.

[2] J. Camargo and S. Macías, On freely decomposable maps, Topology Appl. 159 (2012), 891-899.

[3] J. J. Charatonik, Monotone mappings and unicoherence at subcontinua, Topology Appl. 33 (1989), 209-215. 
[4] J. J. Charatonik, On feebly monotone and related classes of mappings, Topology Appl. 105 (2000), 15-29.

[5] J. J. Charatonik, A. Illanes and S. Macías, Induced mappings on the hyperspaces $\mathcal{C}_{n}(X)$ of a continuum $X$, Houston J. Math. 28 (2002), 781-805.

[6] J. J. Charatonik and P. Pellicer-Covarrubias, On covering mappings on solenoids, Proc. Amer. Math. Soc. 130 (2001), 2145-2154.

[7] W. J. Charatonik, Arc approximation property and confluence of induced mappings, Rocky Mountain J. Math. 28 (1998), 107-154.

[8] J. B. Fugate and L. Mohler, Quasi-monotone and confluent images of irreducible continua, Colloq. Math. 28 (1973), 221-224.

[9] K. R. Gentry, Some properties of the induced map, Fund. Math. 66 (1969/1970), $55-59$.

[10] G. R. Gordh, Jr. and C. B. Hughes, On freely decomposable mappings of continua, Glas. Mat. Ser. III 14(34) (1979), 137-146.

[11] H. Hosokawa, Induced mappings on hyperspaces, Tsukuba J. Math. 21 (1997), 239250 .

[12] A. Illanes and S. B. Nadler, Jr., Hyperspaces. Fundamentals and recent advances, Marcel Dekker, New York, 1999.

[13] K. Kuratowski, Topology. Vol II, Academic Press, New York-London, 1968.

[14] S. Macías, Topics on continua, Chapman \& Hall/CRC, London, New York, Singapore, 2005.

[15] T. Maćkowiak, Continuous mappings on continua, Dissertationes Math. (Rozprawy Mat.) 158 (1979), 1-95.

[16] S. B. Nadler Jr., Hyperspaces of sets, Unabridged edition of the 1978 original, Sociedad Matemática Mexicana, México, 2006.

\section{J. Camargo}

Escuela de Matemáticas, Facultad de Ciencias

Universidad Industrial de Santander

Ciudad Universitaria, Carrera 27 Calle 9, Bucaramanga, Santander, A. A. 678

Colombia

E-mail: jcam@matematicas.uis.edu.co

S. Macías

Instituto de Matemáticas

Universidad Nacional Autónoma de México

Circuito Exterior, Ciudad Universitaria, México D. F., C. P. 04510

Mexico

E-mail: sergiom@matem.unam.mx

Received: 10.7.2012.

Revised: 29.1.2013. 